\title{
Extramedullary hematopoiesis: Elucidating the function of the hematopoietic stem cell niche (Review)
}

\author{
KOUHEI YAMAMOTO ${ }^{1}$, YUKAKO MIWA ${ }^{2}$, SHIHO ABE-SUZUKI ${ }^{1}$, SHINYA ABE $^{1}$, \\ SUSUMU KIRIMURA $^{1}$, IICHIROH ONISHI ${ }^{1}$, MASANOBU KITAGAWA ${ }^{1}$ and MORITO KURATA ${ }^{1}$ \\ ${ }^{1}$ Department of Comprehensive Pathology, Graduate School of Medical and Dental Sciences, \\ Tokyo Medical and Dental University, Bunkyo-ku, Tokyo 113-8519; ${ }^{2}$ Department of Pathology, \\ Kanazawa Medical University Hospital, Kanazawa, Ishikawa 920-0293, Japan
}

Received May 6, 2014; Accepted February 11, 2015

DOI: $10.3892 / \mathrm{mmr} .2015 .4621$

\begin{abstract}
Extramedullary hematopoiesis (EMH) occurs under various circumstances, including during embryonic/developmental periods, pathological status secondary to insufficient bone marrow function or ineffective hematopoiesis, in hematological disorders, for example malignancies, as well as stromal disorders of the bone. EMH is characterized by hematopoietic cell accumulations in multiple body locations. Common EMH locations observed in clinical and pathological practice include the spleen, liver, lymph nodes and para-vertebral regions. Among the various organs associated with $\mathrm{EMH}$, the spleen offers a unique site for evaluation of hematopoietic stem cell (HSC)/niche interactions, as this organ is one of the most common sites of EMH. However, the spleen does not have a major role in embryonic/developmental hematopoiesis. A recent study by our group revealed that circulating HSCs may be trapped by chemokine (C-X-C motif) ligand 12 (CXCL12)-positive cells at the margin of sinuses near CXCL12-positive endothelial cells, resulting in the initiation of the first step of EMH, which is a similar mechanism to bone marrow hematopoiesis. The present review briefly discusses the environment of EMH in extramedullary spaces in order to investigate the mechanisms underlying HSC maintenance, and aid the elucidation of the niche-stem cell interactions that occur in the bone marrow.
\end{abstract}

Correspondence to: Dr Morito Kurata or Professor Masanobu Kitagawa, Department of Comprehensive Pathology, Graduate School of Medical and Dental Sciences, Tokyo Medical and Dental University, 1-5-45 Yushima, Bunkyo-ku, Tokyo 113-8519, Japan

E-mail:kurata.pth2@tmd.ac.jp

E-mail: masa.pth2@tmd.ac.jp

Key words: extramedullary hematopoiesis, spleen, chemokine (C-X-C motif) ligand 12, niche, hematopoietic stem cell

\section{Contents}

1. Introduction

2. Sites of EMH

3. Embryonic/developmental EMH

4. EMH associated with hematological disorders

5. EMH associated with stromal abnormalities

6. Microenvironment of EMH

7. Conclusion

\section{Introduction}

Extramedullary hematopoiesis (EMH) is characterized by the formation and development of blood cells, external to the bone marrow medullary spaces. However, this condition has not been identified physiologically in adults. Therefore, the pathophysiological variations in hematopoietic stem cells (HSCs) and their microenvironment may be a component of the homing, proliferation and maturation processes associated with hematopoietic cells in the extramedullary organs of adults; although, there is currently a dearth of studies addressing the prevalence and fundamental processes of EMH (1). This may be explained by the fact that EMH is generally identified as a secondary or accessory event to another disorder, including benign hematologic disorder (2) or cancer (3), in certain cases developing independently or lacking apparent trigger and without clinical or diagnostic implications $(4,5)$.

In bone marrow, the HSC niche, a distinct microenvironment, maintains the characteristics of HSCs (6). It was recently demonstrated that niche-constituting cells within human bone marrow include critical elements, for example mesenchymal stromal cells (MSCs), blood vessels, perivascular cells, macrophages and endosteal cells (7). Investigations into the various mechanisms underlying the interaction between these elements and HSCs have also been conducted. HSCs occur in the vicinity of, but not necessarily in direct contact with, osteoblastic bone-lining cells that constitute the endosteal niche regions. However, the sinusoidal endothelium also interacts with HSCs, and form the vascular niche (6). Initially it was suggested that the endosteal and vascular niches may control distinct HSC populations; however, more recent data 
suggests that their effects may be more complicated due to functional crosstalk between cells within the two regions $(8,9)$. Furthermore, the vascular niche has been suggested to regulate HSCs in a distinct mechanism or potentially harbor HSCs with various self-renewable properties (10-12).

Certain mesenchymal cells, including chemokine (C-X-C motif) ligand 12 (CXCL12)-abundant reticular cells (CAR cells) (13), nestin-expressing cells (14) and non-myelinating Schwann cells derived from the neural crest (15), have been reported to function as bone marrow niches. Of particular significance in localizing and retaining HSCs in the vicinity of niches are the interactions between CXCR4 and HSCs or CXCL12 (SDF-1). In addition, upregulation of vascular cell adhesion molecule-1 (VCAM-1) and very late antigen-4 (VLA-4) expression (16) may be induced by chemokine interactions through CXCL12. Furthermore, CXCL12-deficient embryos exhibited a marked decrease in HSC populations and hematopoietic function was inhibited; therefore indicating that $\mathrm{CXC12}$ has a critical role during the early stages of bone marrow hematopoietic cell colonization (17).

CXCL12 may potentially be involved, not only in hematopoietic cell colonization, but also in the migration and maintenance of HSCs during EMH. For example, it was demonstrated that stromal cells express CXCL12 and induce B-cell migration in the spleen (18). Recently, in a study by our group, whether CXCL12-positive cells were able to be identified and if they had a role in hematopoietic regions of the spleen during EMH was determined by investigating CXCL12 messenger (m)RNA expression levels and localization of CXCL12-positive cells in the spleen (19). A previous study suggested that hematopoiesis in bone marrow cannot be maintained by a vascular niche alone, as indicated by osteoblast deficient mice (20). Since splenic EMH exhibits neither osteoblastic nor endosteal niches, elucidation of the details of crosstalk between HSCs and the niche and/or microenvironments in splenic EMH may be significant in understanding the overall biology of hematopoiesis in terms of niche.

\section{Sites of EMH}

Spleen. The spleen is a frequent site of EMH during the postnatal period; although, it is only a minor factor in the embryonic stages of hematopoiesis. Despite the fact that the splenic microenvironment is characterized by hypoxic/acidic conditions and numerous macrophages that are harsh and inhospitable for HSCs, EMH usually occurs within the red pulp (21). The mechanisms underlying the interaction between HSCs and the stromal cells of the spleen during EMH will be discussed subsequently.

Liver. Hepatic EMH is normal in infants up to $\sim 5$ weeks of age (22). Conversely, hepatic EMH in adults is associated with various pathological states, including hepatic disorders, sepsis, transplantation, hepatic tumors, for example hepatoblastoma; adenomas and hepatocellular carcinomas $(2,23,24)$. EMH is most often observed within the hepatic sinusoids.

Lymph nodes. As with the spleen or liver, EMH may occasionally occur in the lymph nodes and is frequently associated with underlying hematopoietic neoplasms, for example myeloproliferative neoplasms (MPNs) (25). Therefore, in adults and infants; a hematologic evaluation, including blood cell counts, peripheral blood smear and potentially a bone marrow aspiration/biopsy should be performed if EMH is identified in the lymph nodes.

Other sites. Other tissues that may be associated with EMH include the heart, fatty tissue, adrenal glands, kidney, periosteum, pleural cavity, para-vertebral regions, intra-spinal tissue, pre-sacral region, nasopharyngeal region, para-nasal sinuses (5) and numerous types of benign/malignant neoplasm (26).

\section{Embryonic/developmental EMH}

The high clinical significance of bone marrow transplantation has attracted researchers to focus on the developmental origin of HSCs and niches (27). The yolk sac, in the initial stages of embryonic development, is the initial site of primordial development in the human hematopoietic system, and occurs in the third week of development (28). Vascular elements are initially formed within the yolk sac, as the mesodermal cells begin to accumulate (29). Subsequently, clusters of primitive mesodermal cells emerge in close association with these vascular structures, termed blood islands, and the mesenchymal cells in these blood islands develop into hematopoietic cells and begin to circulate on approximately day 20 of development (30). The development of the rudimentary liver occurs simultaneously to these events (29). HSCs are not generated directly, but instead circulating hematopoietic precursor cells populate the fetal liver (29). These cells proliferate and differentiate in the hepatic parenchyma, forming the primary site of hematopoiesis on approximately day 30 (29).

The aorta-gonads-mesonephros region also produces HSCs. Bone marrow hematopoietic elements commence formation at approximately eight weeks of development, which is relatively late in embryogenesis (30). By week 11, HSCs and the bone marrow niche environment are established in the marrow cavity (30). By contrast, the spleen performs a minor function as a site of erythropoiesis (26). At each stage of development, stromal cell/HSC interactions via cytokines, chemokines and growth factors have significant roles in the maintenance of normal hematopoiesis (31).

\section{EMH associated with hematological disorders}

Expansion of the hematopoietic space outside of the bone marrow has been observed in numerous benign hematological disorders, including thalassemia, sickle cell anemia, hereditary spherocytosis, congenital dyserythroblastic anemia and idiopathic thrombocytopenic purpura (26).

In response to reduced red blood cell (RBC) numbers, a homeostatic mechanism induces an increase in the synthesis of RBCs, typically via the production of erythropoietin (26). If the reduction in RBCs is sufficiently severe, hematopoiesis will occur in the extramedullary spaces (26). If granulocyte or platelet production is also insufficient, a similar state occurs (26).

Notonly benign conditions, but alsoneoplastic disorders, have been described by the term EMH (32-34). Neoplastic myeloid 

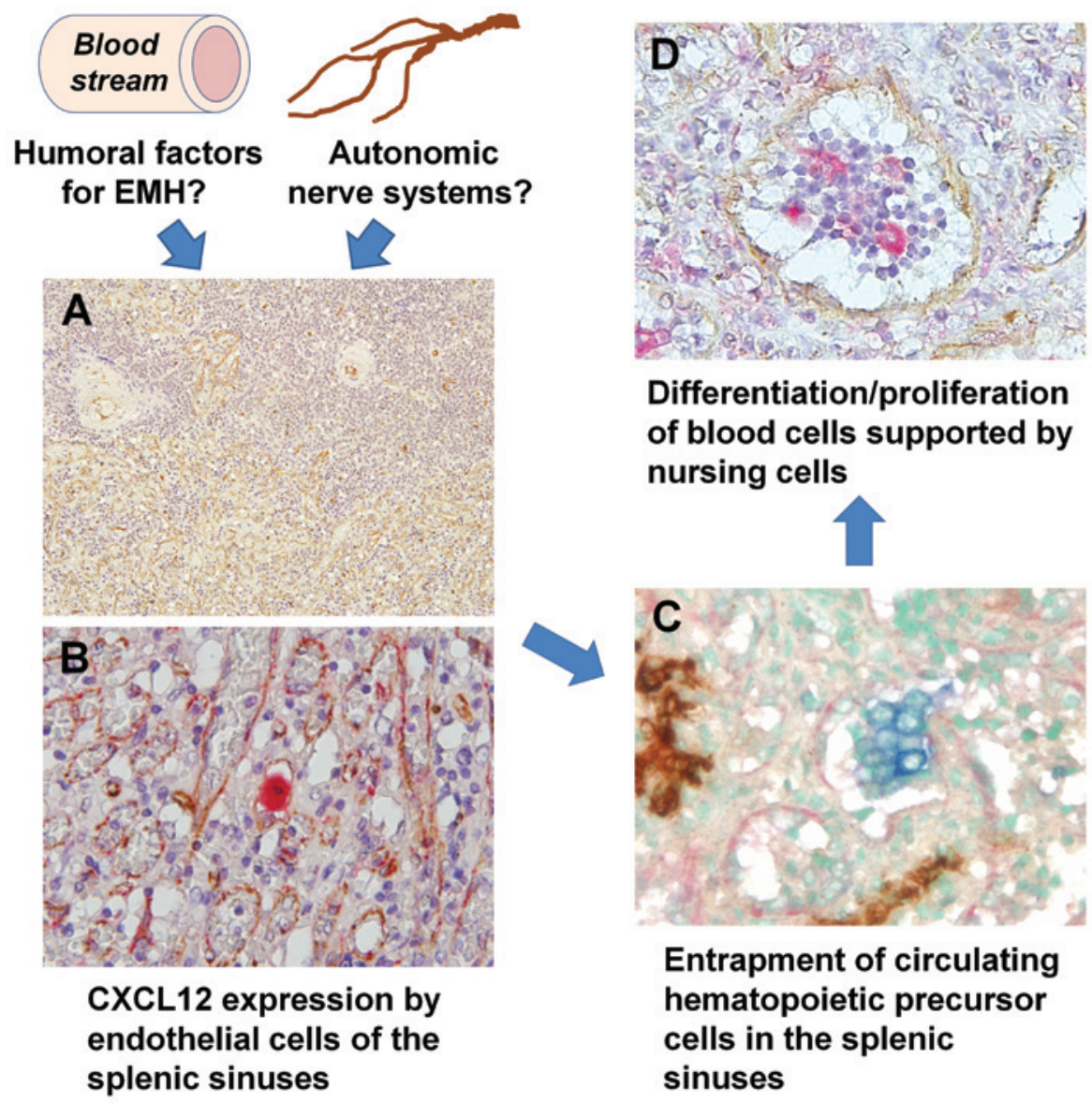

Figure 1. Summary of the distribution of EMH-associated molecules in the spleen. Humoral factors and/or autonomic nerve systems may induce the expression of CXCL12 in splenic sinus endothelial cells. These cells may maintain HSCs, and certain HSCs are able to differentiate into various lineage cells, which are subsequently maintained by macrophages in the sinuses. (A) Localization of CXCL12 (brown) in EMH-positive spleens (single immunohistochemistry; magnification, x50). Note the positive reaction in the margin of the sinuses of the red pulp and arterioles of the white pulp. (B) CXCL12-positive (brown) cells were also positive for factor VIII (red), which was used as a sinus endothelial cell marker, indicating that these cells were sinus endothelial cells (double immunostaining; magnification, x200). (C) c-kit-positive (blue) and CD34-negative (brown) hematopoietic precursor cells were observed adjacent to the CXCL12-positive (red) sinus endothelial cells (triple immunostaining; magnification, x200). (D) Erythroblasts and CD68-positive (red) macrophage-lineage cells were identified in the sinuses of EMH-positive spleens. CXCL12-positive endothelial cells (brown) existed separately from the focus of erythroblastic accumulation (double immunostaining; magnification, x200). CXCL12, chemokine (C-X-C motif) ligand 12; EMH, extramedullary hematopoiesis; HSCs, hematopoietic stem cells.

proliferation in the extramedullary spaces has been identified in association with MPNs, myelodysplastic syndromes, granulocytic sarcoma and other myeloid neoplasms (32-34). Cellular proliferation may consist of trilineage marrow elements similar to those of benign EMH (32-34). The most common sites of EMH associated with neoplastic disorder are the spleen, lymph nodes, skin, bone, small intestine, orbit, breast, cervix, nasal sinus, mediastinum and brain $(26,28,35)$.

\section{EMH associated with stromal abnormalities}

In the infrequent case of stromal disorders of the bone, irregularities in the bone marrow environment trigger EMH (1). Osteopetrosis is an example of such a case, where the marrow cavity is compromised due to excess bone formation and proliferation, resulting in insufficient space for the required hematopoietic element (36). In severe renal osteodystrophy and Paget's disease similar transformations have also been observed (26).

Additionally, EMH is prompted by the replacement of bone marrow cavity spaces with non-hematopoietic and non-osteoid tissues (35). For example, EMH occasionally occurs as a result of extensive involvement of infectious diseases, marrow fibrosis following inflammation, storage diseases including Gaucher and Niemann-Pick diseases or tumor metastasis (37).

\section{Microenvironment of EMH}

Of the various organs associated with $\mathrm{EMH}$, the spleen offers a unique site for evaluating $\mathrm{HSC} /$ niche interactions as it is one of the most common sites of EMH, but does not have a major role in embryonic/developmental hematopoiesis. In the human spleen, it was verified that the expression levels of CXCL12, one of the candidate markers of bone marrow niche-constituting cells, were significantly higher in EMH-positive cases, compared with those in EMH-negative cases (Fig. 1) (19). Furthermore, CXCL12 was demonstrated to be localized to the sinus endothelial cells of the red pulp in EMH-positive spleens; whereas, CXCL12 was expressed throughout the vascular endothelial cells of the white pulp in spleens of EMH-negative and -positive cases (19). EMH frequently occurs in the red pulp, and the data presented in the current 
review suggest that splenic sinus endothelial cells expressing CXCL12 may contribute to the attachment and recruitment of circulating hematopoietic precursor cells, forming bone marrow niche-like regions of EMH in the human spleen (19).

Splenic EMH, in particular erythroid EMH, is suggested to occur within the red pulp of the spleen (39). A study confirmed that erythroblasts, myeloid cells and megakaryocytes were identifiable within the splenic cords and intrasinusoidal spaces in the red pulp of the spleen in EMH-positive cases (21). The identification of multiple stages of differentiation and mitotic erythroblast figures further supported the hypothesis that erythroid EMH occurred in the red pulp (21).

Erythroblastic islands of bone marrow constitute the basic structure required for erythropoiesis, and include central macrophages that form the microenvironment required for erythroblast development and enucleation, as well as phagocytosis of extruded nuclei $(40,41)$. Following the release of HSCs from bone marrow niches, erythroid cell maturation is facilitated and the resulting erythroid colony-stimulating units may adhere to the central macrophages forming distinct erythroblastic islands that are separate from the existing niches (41). This hypothesis was found to be applicable to EMH-positive spleens, where CXCL12-positive signals on endothelial cells were identified distant from clusters of mature erythroblasts (21).

A small population of c-kit-positive/CD34-negative cells have been identified adjacent to CXCL12-positive sinus endothelial cells in the EMH-positive spleen (21). The CD34 antigen has previously been used to identify immature HSCs; however, c-kit-positive/CD34-negative cells have also been shown to comprise a fraction of HSCs (42) or hematopoietic precursor cells, including erythroid progenitors (43). For this reason, c-kit-positive/CD34-negative cells in the EMH-positive spleen may potentially be a type of hematopoietic precursor cell. Bone marrow-derived HSCs in the arterial blood should enter into an open blood system comprised of the red pulp of the splenic cords, so that maturing cells may infiltrate into the venous sinuses $(26,44-48)$. Previous studies have reported the presence of bone marrow-derived HSCs within the sinuses and adjacent to endothelial cells of the spleen $(5,49)$. These results indicate that circulating HSCs may be trapped by CXCL12-positive cells at the sinus margins proximal to CXCL12-positive endothelial cells, in order to initiate the first stage of EMH.

In order for EMH to develop, a niche environment must be activated and expanded by multiple factors, including bone marrow failure, excessive hematopoietic stimulation and tissue inflammation, injury and repair, as well as abnormal cytokine production $(7,26)$. It has been demonstrated that treatment with granulocyte-colony stimulating factor and granulocyte macrophage colony-stimulating factor induces an increase in spleen size associated with EMH $(50,51)$. The underlying causes of $\mathrm{EMH}$ in the spleen were not able to be distinctly classified into the categories, described above; however, further study is required in order to elucidate the mechanisms underlying the upregulation of CXCL12 expression in the spleen.

In addition, distinct tissue niches may induce the development of various subsets of hematopoietic cells from HSCs. A study has suggested that splenic niches may support the development of certain distinct, dendritic-like antigen-presenting cells (52). Although such experiments utilizing animal models have provided useful results and hypotheses, further studies should be conducted in order to identify specific differences in the characteristics and differentiation patterns of HSCs between human bone marrow and splenic EMH.

Bone marrow-derived mesenchymal stromal/stem cells (MSCs) are non-hematopoietic cells with the ability to differentiate into various types of cell, including osteoblasts, adipocytes and chondrocytes. MSCs are known to participate in the formation of niches for HSC development in the bone marrow. Several markers, including CD106, CD166, CD105, CD73 and CD44, are known to be useful in the isolation of MSCs from various organs, including bone marrow, placenta, adipose tissue, synovial membrane, endometrium and cartilage (53). The construction of niche-like regions during EMH may be attributed to the MSC-derived cells of EMH organs, although CXCL12-positive cells were only identified in the red pulp of EMH-positive sinus endothelial cells (19).

\section{Conclusion}

In contrast to how endothelial and perivascular cells and autonomic nerve systems maintain HSCs in the bone marrow $(11,54)$ and how endosteal niches may maintain lymphoid progenitor cells (55),CXCL12-positive endothelial cells in the EMH-positive spleen may only contribute to the localization of HSCs to EMH-positive organs $(5,56,57)$. As the results of a previous study indicated, CXCL12 is expressed in the sinus endothelial cells of the EMH-positive spleen, which may function in the entrapment of circulating hematopoietic precursor cells, whereas the cluster of macrophage/differentiated hematopoietic cells is separated from the CXCL12-positive endothelial cells (19). Other types of cell, including macrophages, as well as nerve, adipose, and dendritic cells, may contribute to the maintenance and differentiation of hematopoietic cells in EMH-positive spleens. CXCL12 was therefore suggested to have a restricted role during EMH, trapping the hematopoietic precursor cells in the sinuses of the splenic red pulp. The function of CXCL12 in bone marrow niche-constituting cells, for example CAR cells, may differ from its function in the EMH-positive spleen. Further studies are required in order to elucidate the mechanisms controlling the EMH niche-like function of various cells in comparison with those of the perivascular and/or endosteal cells of bone marrow niches.

\section{References}

1. Sohawon D, Lau KK, Lau T and Bowden DK: Extra medullary haematopoiesis: a pictorial review of its typical and atypical locations. J Med Imaging Radiat Oncol 56: 534-538, 2012.

2. Tsamandas AC, Jain AB, Raikow RB, Demetris AJ, Nalesnik MA and Randhawa PS: Extramedullary hematopoiesis in the allograft liver. Mod Pathol 8: 671-674, 1995.

3. Vassiliou V, Papamichael D, Lutz S, Eracleous E, Kountourakis P, Polyviou P, Michaelides I, Shoukris M and Andreopoulos D: Presacral extramedullary hematopoiesis in a patient with eectal adenocarcinoma: report of a case and literature review. J Gastrointest Cancer Feb 10, 2012 (Epub ahead of print).

4. Macki M, Bydon M, Papademetriou K, Gokaslan Z and Bydon A: Presacral extramedullary hematopoiesis: an alternative hypothesis. J Clin Neurosci 20: 1664-1668, 2013.

5. Johns JL and Christopher MM: Extramedullary hematopoiesis: a new look at the underlying stem cell niche, theories of development and occurrence in animals. Vet Pathol 49: 508-523, 2012. 
6. Mendelson A and Frenette PS: Hematopoietic stem cell niche maintenance during homeostasis and regeneration. Nat Med 20: 833-846, 2014

7. Flores-Figueroa E, Varma S, Montgomery K, et al: Distinctive contact between CD34+hematopoietic progenitors and CXCL12+CD271+mesenchymal stromal cells in benign and myelodysplastic bone marrow. Lab Invest 92: 1330-1341, 2012.

8. Lilly AJ, Johnson WE and Bunce CM: The haematopoietic stem cell niche: new insights into the mechanisms regulating haematopoietic stem cell behaviour. Stem Cells Int: 274564, 2011.

9. Lo Celso C, Fleming HE, Wu JW, et al: Live-animal tracking of individual haematopoietic stem/progenitor cells in their niche. Nature 457: 92-96, 2009.

10. Kiel MJ and Morrison SJ: Uncertainty in the niches that maintain haematopoietic stem cells. Nat Rev Immunol 8: 290-301, 2008

11. Ding L, Saunders TL, Enikolopov G and Morrison SJ Endothelial and perivascular cells maintain haematopoietic stem cells. Nature 481: 457-462, 2012

12. Levesque JP and Winkler IG: Hierarchy of immature hematopoietic cells related to blood flow and niche. Curr Opin Hematol 18: 220-225, 2011

13. Nagasawa T, Omatsu Y and Sugiyama T: Control of hematopoietic stem cells by the bone marrow stromal niche: the role of reticular cells. Trends Immunol 32: 315-320, 2011.

14. Mendez-Ferrer S, Michurina TV, Ferraro F, et al: Mesenchyma and haematopoietic stem cells form a unique bone marrow niche. Nature 466: 829-834, 2010

15. Yamazaki S, Ema H, Karlsson G, et al: Nonmyelinating Schwann cells maintain hematopoietic stem cell hibernation in the bone marrow niche. Cell 147: 1146-1158, 2011.

16. Avecilla ST, Hattori K, Heissig B, et al: Chemokine-mediated interaction of hematopoietic progenitors with the bone marrow vascular niche is required for thrombopoiesis. Nat Med 10: 64-71, 2004.

17. Ara T, Tokoyoda K, Sugiyama T, et al: Long-term hematopoietic stem cells require stromal cell-derived factor-1 for colonizing bone marrow during ontogeny. Immunity 19: 257-267, 2003.

18. Ellyard JI, Avery DT, Mackay CR, et al: Contribution of stromal cells to the migration, function and retention of plasma cells in human spleen: potential roles of CXCL12, IL-6 and CD54. Eur J Immunol 35: 699-708, 2005.

19. Miwa Y, Hayashi T, Suzuki S, Abe S, Onishi I, Kirimura S, Kitagawa $\mathrm{M}$ and Kurata M: Up-regulated expression of CXCL12 in human spleens with extramedullary haematopoiesis Pathology 45: 408-416, 2013.

20. Visnjic D, Kalajzic Z, Rowe DW, et al: Hematopoiesis is severely altered in mice with an induced osteoblast deficiency. Blood 103 3258-3264, 2004.

21. Wolf BC and Neiman RS: Hypothesis: splenic filtration and the pathogenesis of extramedullary hematopoiesis in agnogenic myeloid metaplasia. Hematol Pathol 1: 77-80, 1987.

22. MacSween RMN, Burt AD, Portmann BC, et al: Pathology of the Liver, 4th ed. Diagn Cytopathol 29: 43, 2003.

23. Schlitt HJ, Schäfers S, Deiwick A, Eckardt KU, Pietsch T, Ebell W, Nashan B, Ringe B, Wonigeit K and Pichlmayr R: Extramedullary erythropoiesis in human liver grafts. Hepatology 21: 689-696, 1995 .

24. Tsamandas AC, Jain AB, Raikow RB, Demetris AJ, Nalesnik MA and Randhawa PS: Extramedullary hematopoiesis in the allograft liver. Mod Pathol 8: 671-674, 1995.

25. Craig CE, Quaglia A and Dhillon AP: Extramedullary haematopoiesis in massive hepatic necrosis. Histopathology 45: 518-525 2004.

26. Sohawon D, Lau KK, Lau T and Bowden DK: Extra-medullary haematopoiesis: a pictorial review of its typical and atypical locations. J Med Imaging Radiat Oncol 56: 538-534, 2012.

27. O'Malley DP: Benign extramedullary myeloid proliferations. Mod Pathol 20: 405-415, 2007.

28. Tavian M, Biasch K, Sinka L, Vallet J and Péault B: Embryonic origin of human hematopoiesis. Int J Dev Biol 54: 1061-1065, 2010

29. Tavian M, Cortés F, Charbord P, Labastie MC and Péault B: Emergence of the haematopoietic system in the human embryo and foetus. Haematologica 84: Suppl EHA-4:1-3, 1999.

30. Georgiades CS, Neyman EG, Francis IR, Sneider MB and Fishman EK. Typical and atypical presentations of extramedullary hemopoiesis. AJR Am J Roentgenol 179: 1239-1243, 2002

31. Tavian $M$ and Péault B: The changing cellular environments of hematopoiesis in human development in utero. Exp Hematol 33: 1062-1069, 2005.
32. Palatnik A, Narayan R and Walters M: Extramedullary hematopoiesis involving uterus, fallopian tubes, and ovaries, mimicking bilateral tuboovarian abscesses. Int J Gynecol Pathol 31: 584-587, 2012.

33. Akbulut S, Yavuz R, Akansu B, Sogutcu N, Arikanoglu Z and Basbug M: Ectopic bone formation and extramedullary hematopoiesis in the thyroid gland: report of a case and literature review. Int Surg 96: 260-265, 2011 .

34. Radopoulos D, Tzakas K and Tahmatzopoulos A: A rare case of renal oncocytoma associated with erythrocytosis: case report. BMC Urol 23: 26, 2006

35. Tavian M and Péault B: Embryonic development of the human hematopoietic system. Int J Dev Biol 49: 243-250, 2005.

36. Neiman RS, Barcos M, Berard C, Bonner H, Mann R, Rydell RE and Bennett JM: Granulocytic sarcoma: a clinicopathologic study of 61 biopsied cases. Cancer 48: 1426-1437, 1981

37. Tavassoli M and Weiss L: An electron microscopic study of spleen in myelofibrosis with myeloid metaplasia. Blood 42: 267-279, 1973

38. YoshidaH,KawaneK,KoikeM,etal:Phosphatidylserine-dependent engulfment by macrophages of nuclei from erythroid precursor cells. Nature 437: 754-758, 2005.

39. Chasis JA and Mohandas N: Erythroblastic islands: niches for erythropoiesis. Blood 112: 470-478, 2008.

40. Sadahira Y and Mori M: Role of the macrophage in erythropoiesis. Pathol Int 49: 841-848, 1999.

41. Sonoda Y and Sasaki K: Surface morphology of the central macrophages of erythroblastic islets in the spleen of aged and pregnant mice: an immunohistochemical light microscopic study. Arch Histol Cytol 71: 155-161, 2008.

42. Sonoda Y: Immunophenotype and functional characteristics of human primitive CD34-negative hematopoietic stem cells: the significance of the intra-bone marrow injection. J Autoimmun 30: 136-144, 2008

43. De Jong MO, Wagemaker G and Wognum AW: Separation of myeloid and erythroid progenitors based on expression of CD34 and c-kit. Blood 86: 4076-4085, 1995

44. Cesta MF: Normal structure, function and histology of the spleen. Toxicol Pathol 34: 455-465, 2006.

45. Mebius RE and Kraal G: Structure and function of the spleen. Nat Rev Immunol 5: 606-616, 2005.

46. Mueller SN and Ahmed R: Lymphoid stroma in the initiation and control of immune responses. Immunol Rev 224: 284-294, 2008.

47. Asakura A and Rudnicki MA: Side population cells from diverse adult tissues are capable of in vitro hematopoietic differentiation. Exp Hematol 30: 1339-1345, 2002

48. Saito H, Yokoi Y, Watanabe S, et al: Reticular meshwork of the spleen in rats studied by electron microscopy. Am J Anat 181: 235-252, 1988

49. Wright DE, Wagers AJ, Gulati AP, et al: Physiological migration of hematopoietic stem and progenitor cells. Science 294: 1933-1936, 2001.

50. Stroncek D, Shawker T, Follmann D and Leitman SF: G-CSF-induced spleen size changes in peripheral blood progenitor cell donors. Transfusion 43: 609-613, 2003.

51. Picardi M, De Rosa G, Selleri C, Scarpato N, Soscia E, Martinelli V, Ciancia R and Rotoli B: Spleen enlargement following recombinant human granulocyte colony-stimulating factor administration for peripheral blood stem cell mobilization. Haematologica 88: 794-800, 2003.

52. O'Neill HC, Griffiths KL, Periasamy P, et al: Spleen as a site for hematopoiesis of a distinct antigen presenting cell type. Stem Cells Int: 954275, 2011.

53. Sivasubramaniyan K, Lehnen D, Ghazanfari R, Sobiesiak M, Harichandan A, Mortha E, Petkova N, Grimm S, Cerabona F, de Zwart P, et al: Phenotypic and functional heterogeneity of human bone marrow- and amnion-derived MSC subsets. Ann NY Acad Sci 1266: 94-106, 2012.

54. Lucas D, Scheiermann C, Chow A, Kunisaki Y, Bruns I, Barrick C, Tessarollo L and Frenette PS: Chemotherapy-induced bone marrow nerve injury impairs hematopoietic regeneration. Nat Med 19: 695-703, 2013.

55. Ding L and Morrison SJ: Haematopoietic stem cells and early lymphoid progenitors occupy distinct bone marrow niches. Nature 495: 231-235, 2013.

56. Chute JP: Stem cell homing. Curr Opin Hematol 13: 399-406, 2006.

57. Jamieson $\mathrm{CH}$, Barroga $\mathrm{CF}$ and Vainchenker WP: Miscreant myeloproliferative disorder stem cells. Leukemia 22: 2011-2019, 2008. 